\title{
Chapter 11
}

\section{Persuasive Health Technology}

Lisette (J.E.W.C.) van Gemert-Pijnen, Saskia Kelders, Nienke Beerlage-de Jong \& Harri OinasKukkonen

\section{Introduction}

This chapter aims to describe persuasive health technology to help you understand the strategies that can improve the adherence to eHealth technologies and increase their effectiveness. As we have seen in Chapter 2, behaviour change is important to improve health and wellbeing. Behaviour change techniques can be implemented in eHealth technology to support users in reaching their goals. Persuasive technology can be seen as the technological instantiation of these behaviour change techniques. Indeed, the technology needs to be persuasive to increase the changes of people using the technology and reaching its health and wellbeing goals. Chapter 10 has shown that methods from Human Centred Design can be employed to make a technology that is appealing and fitting with the users. In this chapter, we will show that technology can do more than only be appealing, as it can also be persuasive, and in this way be an excellent supporter for users to reach their own goals. The chapter starts with an introduction of persuasive technology and how this has been applied in the context of improving health and wellbeing. We introduce the Persuasive Systems Design (PSD) model and how it can be used to develop and evaluate eHealth technologies. After completing this chapter, you will be able to:

- explain what persuasive technology is, and in what way domains such as persuasive communication, health promotion, social marketing, technology acceptance and human-media interaction are underlying foundations.

- analyse the added value of persuasive technology in the context of improving health

- explain the PSD model, name the four categories, and provide examples of accompanying persuasive features.

- explain in what way persuasive technology can be used to develop and evaluate eHealth technologies.

- provide examples of how persuasive features can be integrated into an eHealth technology.

\section{What is persuasive technology?}

As stated in the introduction, technology can try to persuade the user to change their behaviour. In the late 1990's, Fogg suggested that this could be called persuasive technology: a field that 
studies any interactive information technologies intentionally designed to change users' attitudes or behaviour. Since then, this field has received growing interest among both researchers and practitioners. Based on Fogg, Oinas-Kukkonen and Harjumaa more recently defined persuasive systems as 'computerized software or information systems designed to reinforce, change or shape attitudes or behaviours or both without using coercion or deception' (Oinas-Kukkonen \& Harjumaa, 2009). Important to note in this definition is that persuasive systems are by definition voluntary. Therefore, while other forms of persuasion, including deception, coercion and monetary inducements may be effective, they are not what we call persuasive technology.

But what makes technology persuasive? Increased interactivity and engagement of users through modern information and communication technologies have opened up many opportunities to influence users' behaviours. In the context of health, web-based interventions have been developed to educate, inform, or treat people to reduce risk behaviours, and to promote a healthier lifestyle. As we have seen in Chapter 3, computing technologies have capacities that distinguish them from human persuaders. Some of these capacities are anonymity, persistence, and the opportunity to control a virtually unlimited storage of data.

Moreover, they can use different cues for communication simultaneously (e.g., text, speech, video, and graphics), and they can have access to situations that human persuaders would not be allowed in (e.g., bathroom) or unable to be in (e.g., systems embedded in clothing) (Fogg, 2002; IJsselsteijn, de Kort, Midden, Eggen, \& van den Hoven, 2006). They can be embedded, for example, in virtual environments that become persuasive environments and stimulate people to change behaviour more effectively than traditional media, such as the case of virtual reality. An example can be found in rehabilitation therapy after a stroke: people can exceed their thresholds for physical behaviour and do exercises they think they are not able to do using virtual environments, such as increasing their range of arm movement. Technology can also be persuasive in its ability to be motivating and fun. Think of video games to improve activity and balance like the WII, Pokémon Go, or virtual reality to overcome phobias, for example, of spiders or heights.

The field of persuasive technology is not limited to eHealth. In many other areas, technology has been designed to change behaviour. For example, in marketing, persuasive technology has been used to increase online sales, and in the ecological fields, it has been used to decrease the energy consumption of individuals. However, the promotion of health and wellbeing is one of the most prominent areas for application of persuasive technology. This is not surprising when looking at some of the opportunities persuasive technology offers to health and wellbeing. For example:

- As it was shown in Chapter 2, improving health often implies changing behaviour. Persuasive technology is designed for this goal and can be seen as the technological instantiation of behaviour change techniques. 
- eHealth technology should often be used more than once, persuasive technology can help motivate people to use the technology more often.

In the remainder of the chapter, when referring to persuasive technology, we refer to persuasive technology in the field of health and wellbeing, briefly defined as persuasive eHealth technology.

\section{The Foundations of Persuasive Technology}

Although the field of persuasive technology is only a few decades old, it is based on various well-researched theories stemming from different areas. These include persuasive communication, health promotion, social marketing, and human-media interaction. The theoretical foundations from these fields are described in the following sections.

\section{Persuasive communication}

Persuasive technology involves communication; people interact with technology. Information is mediated through a technology and its users. Persuasive communication intends to describe, explain, and predict the factors that contribute to change attitudes and behaviours (Ajzen, 1992; Dillard \& Pfau, 2002; Perloff, 1993). For example, persuasive communication describes and explains the four layers of communication that influence the understanding of information (Schulz von Thun, 1981). These are (1) the factual layers which refers to facts, data; (2) the expressive layer referring to the relationship between sender and receiver; (3) the self-revealing layer, expressing something about the sender's emotions, values etc.; and (4) the appellation layer referring to the desire, advice, instructions, and effects.

The model by Schulz von Thun has been used widely to describe and explain miscommunications in business, as well as in treatment settings, to improve relationships. The four layers of the communication model are part of the process of persuasion, since the four aspects influence the coding (sending) and decoding (receiving) of information. The way this coding and decoding is done, grounds theoretical models about health communication to promote public health (e.g., infectious diseases campaigns) or to promote a healthier lifestyle in chronic care (Kreps, Bonaguro, \& Query Jr, 2003; Kreps \& Maibach, 2008; Rimal \& Lapinski, 2009). In the field of social psychology, the communication model has been used to further describe and explain the mediating processes of information exchange, by searching for characteristics that play a role in communication and how these characteristics influence each other. Some of these influential characteristics are the sender, receiver, messages, channels, and the settings. In particular the processes of getting attention, comprehension of information, and acceptance of information have been studied in the area of persuasive communication to change attitudes and behaviours (McGuire, 1985). 
Health promotion

In health promotion the Theory of Planned Behaviour (TPB) (Ajzen, 1991; Fishbein, 1979) and the Elaboration Likelihood model (ELM) (Petty \& Cacioppo, 1986) were dominant in researching the persuasiveness of communication. The concepts of the Theory of Planned Behaviour and the Elaboration Likelihood Model (see also Chapter 2) are used in persuasive technology to understand and influence how people act. The TPB assumes that intention is the most important predictor of (intentional) behaviour, and that it is influenced by attitudes, perceived behavioural control, and subjective norm. The ELM explains how attitudes are being changed via several pathways of information processing: ranging from the very conscious central route, and the more indirect, less conscious peripheral route. From these theories, the central hypothesis is that the success of a persuasion attempt depends on the social context, beliefs and norms, and the way in which information is processed by the receiver. The uptake of information is influenced by these internal and external factors, like the motivation and capability of the receiver to process the message. This body of research has addressed issues as low interest and low motivation, and has shed light on some of the needed capacities of a sender to influence the effectiveness of communication (e.g., credibility, attractiveness). For instance, these can be measured as understanding or acceptance of information, or a change in attitude of the receiver.

The Elaboration Likelihood Model (Petty \& Cacioppo, 1986) is of specific interest to persuasive technology researchers as it states that there are two routes for persuading people. The central route underscores reason and argument. The peripheral route builds upon social cues and often on the way arguments are provided, instead of the quality and content of arguments. This peripheral route takes into account contextual (peripheral) factors and the cognitive processing of information, the motivations and abilities to understand messages. This way, the ELM challenges persuasive technology designers to not only focus on giving information and try to persuade people using the central route, but also to formulate strategies to trigger motivations and to increase the capabilities to understand information, specifically for working with technology.

\section{Social marketing}

In social marketing the focus is on marketing principles and compliance strategies to influence a target audience to voluntarily accept, reject, modify, or abandon a behaviour for the benefit of individuals, groups, or society as a whole (Kotler, Roberto, \& Lee, 2002). These principles are often used as techniques to persuade. The basic principles from social marketing are social norms, conformity, and compliance to realize goal-directed behaviours. Social norms refer to rules and standards that are understood by members of a group, that guide and/or constraint social behaviour without the force of laws. Conformity refers to the willingness of people to conform to others because of the social benefits of being accepted by them. Goal-directed 
behaviours are, for example, to behave effectively, to build and maintain relationships, or to develop their self-concept. Principles of compliance, which increase the chance of people complying with a suggestion or request, are (Cialdini, 2001):

- Reciprocity: people feel obligated to return a favour

- Scarcity: when something is scarce, people value it more

- Authority: people are more inclined to comply with authorities

- Commitment and constancy: people do as they said they would

- Consensus: people do what others do

- Liking: people say yes to people they like

Acceptance and human-media interaction

Most theories above focus on the persuasion-aspect of persuasive technology. The technological aspect remains underexposed in these theories. Two additional approaches provide theories specifically focused on the technological aspects: acceptance of technology and human media interaction.

Theories of acceptance of technology are mainly based on cognitive psychology. They are used to investigate and influence predictors for acceptance of or adherence to technologies, especially web-based interventions for health promotion. The Technology Acceptance Model (TAM and TAM 2;(Davis, 1985; Venkatesh \& Davis, 2000) and Unified Theory of Use and Acceptance (UTAUT; (Venkatesh, Morris, Davis, \& Davis, 2003) have their roots in these theories.

Important aspects that influence the intentions to use a certain technology from these models are perceptions about ease of use, performance and effort expectancies, and social influence. These perceptions are assumptions people have about whether they are able to use a technology and they can benefit from that use. Although perceptions are important to fine-tune a technology to peoples reported expectations, in practice this might not predict actual use of a technology. Perceptions and expectations might be a good predictor for the intention to use a technology, but when there are barriers that hinder actual use, these intentions may remain intentions only (see Chapter 13 and (Nijland, van Gemert-Pijnen, Kelders, Brandenburg, \& Seydel, 2011).

Theories on human-media interaction and computer-mediated communication are used to investigate how people interact with and are influenced by information technology. These fields cover, for example, the design of interfaces to interact with technology and how technology can support people to perform tasks, to do exercises, or to communicate with others. Chapter 10 on human centred design provides some examples of methods used in these domains.

\section{Developing and Evaluating Persuasive Technology}


To develop unobtrusive technologies that facilitate the achievement of peoples' goals, a deep understanding is needed of how people interact with and are influenced by technology. As we have seen, this requires an interdisciplinary approach, for example, using persuasive communication, social psychology, engineering, and human centred design.

This multidisciplinary approach is not a 'one-way street' in that, for instance, theories on persuasive communication and social psychology can only be used to inform the design and understand the influence of persuasive technology. The use of technologies as persuaders can also shed a new light on the interaction-process of persuasion and refine existing theories. Within persuasive technology, the interaction takes place between a system and different users (known or unknown), where the system can be seen as the 'representative' of the designers of a certain technology. Therefore, computer-human persuasion is more complex than a traditional senderreceiver interaction via face to face or text, because of an additional influential medium: the technology. Although human-computer interactions are social in nature and people see computers as social actors, it is rather unknown how these interactions re-shape attitude, beliefs, and emotions, or how they actually change behaviour. As humans interact with technologies, the meaning of a certain technology can change over time. For example, in most cases users employ technologies in different ways to change their attitude and behaviour. They can, for example, monitor their behaviour and quantify themselves to manage a disease such as diabetes. Thus, they can retrieve and add information to a device, they communicate about the results (e.g., via e-mail), and they receive feedback. In such a way, users re-shape a technology and may change their goals as they use it.

This means that persuasion is not a static ad-hoc event but an ongoing process. This requires behaviour models and techniques that are context and process driven, as opposed to more static models such as the Theory of Planned Behaviour or the Technology Acceptance Model. Issues of persuasive design will become a critical area of focus within eHealth intervention development and will have considerable effects on usage and outcome. The employment of persuasive strategies into the design can increase the adherence to interventions, and its desired effects to be achieved (Kelders, Kok, Ossebaard, \& Van Gemert-Pijnen, 2012; Wildeboer, Kelders, \& van Gemert-Pijnen, 2016). Yet, theoretical frameworks and conceptual models are needed that pay closer attention to mapping the lessons learned from psychological studies, with the characteristics of modern information and communication technologies.

As we saw in Chapter 7, persuasive technology is integrated in the CeHRes Roadmap to design technologies that are user-friendly, and that motivate and engage users to change their attitudes and behaviours. In particular, the Persuasive Systems Design (PSD) model (Oinas-Kukkonen \& Harjumaa, 2009) plays an important role in the application of persuasive features to increase engagement and adherence to technologies, and with the intent to change behaviours. The PSD model provides the framework to decide and test what kind of features could be applied within a 
persuasive eHealth technology, depending on, for example, the target group and desired goals to be achieved. As such, it is connected to the design stage of the CeHRes Roadmap.

\section{The Persuasive Systems Design model}

Fogg's seminal book 'Persuasive Technology' (2002) was the first suggested conceptualization for helping software designers to create technology that can influence its users. Fogg states that, for its users, information technology can be persuasive in the role of a tool (e.g., making a task easier), a medium (e.g., providing an experience), or a social actor (e.g., levering principles from social influence). The PSD model is a more recent and state-of-the-art approach for designing and evaluating persuasive systems. The PSD (Oinas-Kukkonen \& Harjumaa, 2009) model defines seven premises or core issues that are common for all persuasive systems:

1) Information technology, at least philosophically, is never neutral, but rather it always influences its user(s) in one way or another. 2) Moreover, being able to build persuasive systems requires insight from both software design and psychology. Lessons learned from psychology include that people like their views about the world to be organized and consistent, 3) persuasion is often incremental, a gradual process of steps, and 4) the direct and indirect routes (ELM) are key persuasion strategies, even though psychological theories tend to differ between each other in their views to these premises. 5) Software design requirements comprise that persuasive systems should be both useful and easy to use, which is much easier said than done, and 6) that persuasion through these systems must always be unobtrusive to a user's primary tasks, and 7) should therefore be transparent.

After obtaining a deeper understanding of persuasion premises, the next step in designing persuasive technology, according to the PSD model, is a careful analysis of the context of persuasion. This includes the targeted users and the intended use of the system, as well as the users' intentions to change behaviours, the environment (locations, etc.), and strategies for persuasion, rooted in aforementioned models and theories to discern opportune and/or inopportune moments for applying persuasive features. Analysing the context for persuasion is needed for all stages of design and evaluation of persuasive systems, ranging from evaluating software specifications in the early stages of systems development, to studying full-fledged commercial applications.

Lastly, the PSD model defines four categories of software features for persuasive systems, namely primary task support, computer-human dialogue support, system credibility support and social support. Thus, different types of persuasive software features, grounded in psychological theories, can be implemented to support the user's primary activities, represent information fluently in the computer-human dialogue, convey the credibility of information being presented, and to leverage social influence. A thorough explanation of all the features is beyond the scope 
of this chapter and can be found in the work of (Oinas-Kukkonen \& Harjumaa, 2009). Table 1 shows the seven features of each category, which are explained in a bit more detail inside Box 1 , and Figure 1 illustrates some of the persuasive features. Important to note is that the seven features in each category are not exhaustive: there are other possible features imaginable that can support each of the four categories. Moreover, the model does not put forward a claim that all imaginable software features should be implemented into a persuasive system, or that more is always better.

\section{<TABLE 11.1 HERE>}

\section{Box 1 - Description of PSD model categories}

Primary task support

Primary Task Support facilitates the performance of the user in carrying out the primary activities to reach the goal of the intervention (e.g., decrease depressive symptoms, improve selfmanagement of diabetes). The purpose of these features is to facilitate to the user the friendliness and understandability of the information, by presenting it in personalized and small steps, reduced to tiny behaviours to achieve their goals, as well as to provide monitoring strategies of users' performance and progress towards such goals.

\section{Dialogue support}

Dialogue support facilitates the interaction between a system and the user by providing persuasive features that aim to motivate and engage the user to achieve desired goals with the system. The dialogue is incentive-driven to motivate users in a positive way, so that the technology takes a social role, for example, an avatar that guides you through exercises.

\section{System credibility support}

Credibility Support refers to the trustworthiness and reliability of the system. With the use of the persuasive features, transparency can be given to the background of the system (expertise, authority, etc.). Users need that information, particularly to decide the verifiability of it.

\section{Social support}

Social Support features motivate users by leveraging social influence of other people. Users can compare themselves with others, such as relatives or unknown people, or share information with those who have the same goal to achieve desired behaviours. 
In this screenshot of the eMental Health intervention 'This is Your Life' (introduced in Chapter 5), several persuasive features can be identified, as is described below.

\section{$<$ FIGURE 11.1 HERE>}

Figure 1. Screenshot from This is Your Life, a gamified intervention to promote wellbeing (Ludden, Kelders, \& Snippert, 2014).

In Figure 1, tunneling can be seen as the route participants take throughout the map. These are the lessons that guide the users through the intervention. The intervention is also somewhat personalized, by providing a picture of the user on the screen. Self-monitoring is present as the user can see the progress she made, visualized by the progress bar/picture on the top of the screen and the 'domes' around some of the areas indicating that after competing these areas the user improved on the outcome measures of that area. Rewards are visible as the badges the user has earned, shown on the right of the screen. Arguably, liking is present due to the whole design of the intervention being attractive to the users. Lastly, social role is present as the avatar of the professor. With this avatar, the system itself adopts a social role, in this case a coaching professor, which makes the system more persuasive.

\section{Research into Persuasive eHealth Technology}

Over the last years, considerable research has been conducted using the PSD-model to understand the impact of persuasive eHealth technology and to improve this impact. The PSD model has been used mainly as an evaluation framework to understand what persuasive features improve adherence and what combinations of features have been used to achieve the desired effects. In this section, we describe how the PSD model has been used as an evaluation framework to give insight into the observed and potential impact of persuasive eHealth technology. We also explain how it can be used as a framework to design eHealth interventions, and what ethical considerations are related to persuasive eHealth technology.

\section{The PSD model as an evaluation framework}

The PSD model has been used to evaluate what kind of features are employed in eHealth technologies, to a lesser extent to measure the effects of these features on adherence and outcomes. To measure what features matter, research has been conducted on the perceived persuasiveness of technologies.

\section{Types of features}

Various studies have shown that within persuasive eHealth technologies, features supporting the Primary Task are most often used and reported compared to the other categories. The other 
categories (dialogue, credibility and social support) vary in their use, depending on the focus and mode of the eHealth technology. For example, within web-based interventions for weight loss, social support was employed widely (Lehto, Oinas-Kukkonen, \& Drozd, 2012; OinasKukkonen, 2013), whereas this was less used in web-based alcohol and smoking (Lehto \& Oinas-Kukkonen, 2011), and was hardly used in web-based mental health interventions (Kelders et al., 2012).

Looking at separate features, especially reduction (primary task support) and reminders (dialogue support) seem to be often employed across the board. Tailoring, adapting an intervention to specific target groups, is employed to a varying degree. Some studies have found this feature to be used very often (Kelders et al., 2012), while other studies have noticed a lack of using this feature (Langrial, Lehto, Oinas-Kukkonen, Harjumaa, \& Karppinen, 2012; Lehto \& Oinas-Kukkonen, 2011; Lehto et al., 2012, Oinas-Kukkonen, 2013). This may also be attributed to the way researchers have studied these features. Often, it is merely assessed whether a feature is present or not, and the quality or extent of the feature is not taken into account. This may entail that even the slightest use of tailoring is interpreted by one researcher as 'present', while others would interpret this as not enough to merit the feature as being present.

An issue that is often reported in these kinds of studies is that the reasons for including (or omitting) specific features are not given. In most cases, the underlying theories or principles for persuasion are not reported (Langrial et al., 2012; Lehto et al., 2012; Oinas-Kukkonen, 2013). Often, no information is given on how these interventions were developed (Kelders et al., 2012), and the persuasive context (intent, event, strategy) for developing the interventions were not specified (Langrial et al., 2012).

\section{Influence on adherence}

Few studies have investigated the relationship between persuasive features and adherence (e.g., using an intervention as intended by the developers, for instance, in terms of duration and features, see Chapter 13). As persuasive technology has the ability to motivate users and change their behaviour, it seems logical to assume that it can consequently influence the way and frequency by which eHealth interventions are used. Persuasive features could be important to increase adherence when the appropriate principles are deployed on those critical moments when non-adherence starts (Kelders \& van Gemert-Pijnen, 2013). A reminder at the right time, or a smart reward, may give the user just a little bit of extra motivation to stick with the program.

This hypothesis is supported by a large systematic review including 83 web-based interventions to improve health and wellbeing (Kelders et al., 2012). The review showed that increased employment of dialogue support features increased the adherence to web-based interventions. Although social support features were hardly used, it seemed that interventions that did employ 
these features more elaborately achieved higher adherence rates. Primary Task Support did not show a significant contribution to adherence. The study showed the importance of a persuasive design of the interaction between the system and its users for sustained use of technology, while also showing that the possibilities to do this are still hardly employed in web-based interventions in the health area.

\section{Influence on effectiveness}

Studies have shown that persuasive systems as a whole tend to be effective in improving health and wellbeing (Hamari, Koivisto, \& Pakkanen, 2014). However, these kinds of studies often compare a persuasive system to no intervention or 'care as usual'. Therefore, no claims can be made about the influence of the persuasiveness of the technology, or which features are most effective. To shed more light on these questions, some studies have investigated the relationship between the number of persuasive features and effectiveness, or between specific features and effectiveness.

It seems that there is a positive relationship between the number of persuasive features and effectiveness (Wildeboer et al., 2016; Xu, Chomutare, \& Iyengar, 2014), suggesting that persuasive technology indeed adds to the effectiveness of eHealth technology. However, it cannot be concluded that the more features are being used, the more effective a technology is. It may be more important to focus on what combinations of features will yield the best possible outcomes. Studies have shown that some PSD features work well together while others don't. Using features that supplement each other can strengthen the persuasive effects. For example, rewards can supplement suggestion, making this an effective combination (Räisänen, Lehto, \& Oinas-Kukkonen, 2011). Also, the combination of tunneling, tailoring, reminders, social learning, social comparison, with or without similarity, seems to contribute to higher effect sizes (Wildeboer et al., 2016). However, it must be avoided to weaken persuasiveness by implementing features that have low synergy. For example, abundant use of reduction makes tunneling redundant (Räisänen et al., 2010).

It seems that it is important to employ features from all the three different categories to be most effective, but more research is needed to verify this hypothesis. Moreover, research is needed to ground the use of features in behaviour change techniques, persuasive communication and social psychology. In most studies, the rationale behind the choice of features and the users' evaluation of the persuasiveness of the eHealth technology is lacking, so it is difficult to draw generalizable conclusions about which features to employ in which contexts.

\section{Perceived persuasiveness}

A different way of studying the influence of persuasive features is to investigate whether a system with these features is perceived as more persuasive by its users, and whether a system 
that is perceived as more persuasive is actually more effective in changing behaviour. In this way, the assumptions that employing persuasive features increases the persuasiveness of the system, and that this increased persuasiveness makes a system more effective, can be tested.

To measure perceived persuasiveness, a questionnaire was developed based on the PSD model, called the Perceived Persuasiveness Questionnaire (PPQ) (Lehto et al., 2012; Oinas-Kukkonen, 2013). Although it has not been validated yet, it has shown to be a promising low-threshold instrument to measure perceived persuasion. However, research indicates that some of the constructs of the PPQ could be adapted to even better fit the PSD model and to fit the mental models of the potential target group (e.g., technology users) (Jong, Wentzel, Kelders, OinasKukkonen, \& Gemert-Pijnen, 2014).

Early research on the concept of perceived persuasiveness indicates that it is related to the intention and actual use of persuasive eHealth technology (Drozd, Lehto, \& Oinas-Kukkonen, 2012; Oinas-Kukkonen, 2013). Especially perceived dialogue support seemed a crucial factor for the persuasiveness of the web-based system, as a 'connecting node to other related factors' (perceived primary task support and perceived credibility support) and perceived persuasiveness. It shows the importance of dialogue: it enables appropriate feedback and counselling, and stimulates the interaction with a system, thereby making it a pivotal aspect in eHealth technologies that provide coaching and support for people to improve their health and wellbeing.

\section{Employing PSD model evaluation outcomes to design persuasive eHealth technology}

Both the PSD model itself and insights from evaluation research have been used as guidelines for the design of persuasive eHealth technologies. For instance, the PSD model itself is intended as a guideline for the design, where developers need to address the premises, the context, and employ primary task, dialogue, credibility and/or social support features to create a persuasive eHealth technology. Also, recommendations on how to design eHealth technologies have come from studies on specific persuasive technology strategies, from theories and theory-based studies. This has led to general design guidelines and guidelines on ways to use a specific feature as 'suggestion' (Andrew, Borriello, \& Fogarty, 2007; Consolvo, McDonald, \& Landay, 2009; Ploderer, Reitberger, Oinas-Kukkonen, \& van Gemert-Pijnen, 2014). Although these studies provide valuable insights, more in-depth work needs to be done on validating and studying the effects of using these design guidelines. Specifically, attention needs to be paid to the ways in which the persuasive design of eHealth technology can improve the usability, adherence, and fit with its users.

\section{Improving usability}


From evaluation research we know that users of eHealth technologies face usability issues: they get lost in interventions (Sieverink, Kelders, Braakman-Jansen, \& van Gemert-Pijnen, 2014), have issues finding exercises that should be done daily (Van Gemert-Pijnen, Kelders, \& Bohlmeijer, 2014), or they do not know what features they should use to reach their goals (Akkersdijk, Kelders, \& Gemert-Pijnen, 2016). Persuasive technology can provide support to help users overcome these usability issues. Dialogue support features seem to be able to provide guidance by, for example, suggesting useful exercises based on the goals a user has entered. Primary Task Support features may be used to guide people through the intervention logic. For example, to support caregivers to register infections in nursing homes, reduction and tunnelling were used to guide the user in the step-by step registration of infections and used antibiotics (Beerlage-de Jong, Eikelenboom-Boskamp, Voss, Sanderman, \& van Gemert-Pijnen, 2014).

These features that can support usability can be designed in the system in advance when usability issues are expected. However, sometimes these issues only surface after a system is

implemented. Log data can provide insight in where and when persuasive features are needed to improve usability (see Chapter 8 and 14). For example, within an eMental Health intervention for people with depressive symptoms, log data revealed that many users occasionally log in to the system without accessing any of the features (Kelders, Bohlmeijer, \& Van Gemert-Pijnen, 2013). This points towards a non-effective usage of the system. Additional investigation found that users use these logins only to see whether they can start the next session. To improve the usability, a persuasive feature such as reminders ('Your new session is ready!') can be used to avoid these unnecessary logins, or a feature as suggestion or rehearsal can be used to make these short sessions more useful (e.g., a message when logging in to the system saying 'Your next session is not yet ready, but it would be very beneficial to do this exercise again.')

\section{Improving adherence}

Persuasive strategies are still relatively new to eHealth design. But attention has increased, as it is now becoming clear that non-adherence to technology based eHealth interventions may partially be explained by unattractive interfaces or obtrusive designs (Ludden, van Rompay, Kelders, \& van Gemert-Pijnen, 2015). Technology often does not motivate or engage users to stick around and complete the program. We know that built-in persuasive features, tailored to a certain user profile, increase adherence. For example, reminders can be used to trigger users to continue use, and social support features can also entice users to return to the intervention (Freyne, Saunders, Brindal, Berkovsky, \& Smith, 2012). A study of Cavanagh and Millings discusses 'common factors' such as generating hope, empathy and warmth, collaboration and feedback, that increase the effectiveness of interventions (Cavanagh \& Millings, 2013). The study provides evidence that these factors may be built into eHealth technologies by using persuasive technology. For example, in therapeutic settings creating a relationship (therapeutic alliance) with a therapist can have a positive effect on adherence to the therapy. In case of self- 
guiding eHealth interventions, a therapist can be a virtual person. Persuasive features can be used to support tasks (e.g., performing exercises), or to support a dialogue with a virtual coach (to express emotions, empathy) (Scholten, Kelders, \& van Gemert-Pijnen, in press). In experimental research, it can be examined what features affect the performance of tasks and emotions during the use of eHealth technology, resulting in a positive relationship with a virtual coach and prolonged adherence.

\section{Improving fit with users}

A last question within the design of persuasive eHealth technology is how to create a technology that fits with the users. As discussed in Chapter 13, this fit can lead to high user-engagement and a good fit can be beneficial to effectiveness, adherence, and implementation in practice. However, until now, not much is known on how to personalize interventions to achieve this fit.

Studies have investigated whether user characteristics as personality, gender and gamer type can be used to match users to persuasive features (Beerlage-de Jong, Wrede, van Gemert-Pijnen, \& Sieverink, 2017; Drozd et al., 2012; Oinas-Kukkonen, 2013; Halko \& Kientz, 2010; Orji, Vassileva, \& Mandryk, 2014). All of these studies have shown that these characteristics impact the effectiveness of different features. They indicate that persuasion might be more effective when tailored to the user rather than implementing a 'one size fits all' version of a technology. However, as there may be many user characteristics that impact the effectiveness of persuasive features, and it may be that the influence of these characteristics varies in different contexts, it seems to be unfeasible to explore all characteristics that influence persuasiveness in all contexts.

Other approaches have tried to overcome this issue by, for example, using the concepts of persuadability and engagement. Kaptein et al. have used questionnaires to create a persuadability score of each individual (Kaptein, Lacroix, \& Saini, 2010). This persuadability score is a measure of the tendency of a person to comply with the different persuasive strategies. The study showed that people with a high persuadability score are more persuaded by health-related messages that employ these persuasive strategies than people with a low persuadability score. Although there are limitations of this study (e.g., it investigated short-term effects on a single behaviour), it is important because it shows that differences in persuadability of people (and therefore in the potential effectiveness of persuasive technology) can be assessed and utilized, at least from a theoretical starting point. In the same way, it has been posited that selecting the persuasive features that invoke the most engagement in each individual may be a way to personalize interventions. Such knowledge can be used to design more effective eHealth interventions and to increase adherence to these interventions.

\section{Ethics}


Ethics are an important part of persuasive technology. An ideal system literally persuades its users to adopt the target behaviour. Computer-mediated persuasion means that people are persuading others through computers, for example, via instant messages or social networking systems. Some patterns of interaction, which are similar to social communication, may be utilized also in computer-human persuasion, even if a technology cannot communicate in the same way humans do. In the case of persuasive systems, there are always stakeholders who have the intention of influencing someone's attitudes or behaviour, because computers do not have intentions of their own, at least at this point in time.

The 'dark sides' of persuasion are manipulation or coercion, forcing people to do something on a non-voluntary base. By definition this is out of scope of in the 'positive' oriented approach of supporting people to behave healthier as is the case with persuasive (eHealth) technology. However, it is imaginable that a certain form of manipulation can be (and is) used to push people in the right modus to control their behaviours. For example, nudging people to support decision making on a healthier lifestyle can be considered as manipulation by providing a limited spectrum of choices (just providing 'good' products or services).

Some of the ethical issues one should think of when developing a persuasive eHealth technology are (Bioethics, 2015):

- Responsibility. Whose responsibility is it that people lead healthy lives? When developing a persuasive eHealth technology to stimulate people to lead more healthy lives, the assumption may be that the developers of the technology have a responsibility in the self-management of people. However, using a persuasive technology and not, for instance, more firm techniques such as rules or legislation, also emphasizes the free choice aspect of behaviour. This in turn might push people towards being wholly responsible for their own wellbeing (or the lack of), while not everyone may be able to deal with this responsibility.

- Autonomy: This is the right of individuals to make their own choices, based on their own values. Persuasive technology may limit autonomy by first deciding on what the desired behaviour is, for example, based on social norms, thus limiting the person's autonomy to choose their own desired behaviour. Second, the technology will also nudge people towards behaving in that specific way, limiting the choice of people behaving in different ways.

- Impact on self-control. It may be that being persuaded to behave a certain way actually limits an individual's self-control in the long term: it may make it even harder to behave in a desired way when not having a specific persuasive eHealth technology to assist him or her. Thus, there is a chance people might become too dependent on technology. 
- Equity: Although technology may make healthcare more accessible to many different people, persuasive eHealth technology may also hinder equity in different ways. First, as these technologies make decisions about certain desired behaviours, they may only reach people that already share these ideas and norms. Second, some of the persuasive techniques might be more effective for different people, therefore influencing them in different ways. For example, people with lower literacy skills may lack the ability to carefully weigh arguments, but may defer to authority more readily, making them more influenced by the use of authority figures.

\section{The Future of Persuasive Health Technology}

Persuasive technology, when aimed to change behaviours in the domain of health and wellbeing, is a promising field. Although we need to have more theoretical insights in what works best for whom, and what the 'dark sides' are from using persuasive features to nudge people to do what we think is best for them. To design health technologies that are usable and that motivate users to improve behaviours, we need more insight in persuasive features to understand and predict factors that improve adherence to and effectiveness of eHealth technologies. More research is needed on, for example:

- Understanding how behaviour change techniques can be used to ground persuasive features (see Chapter 2). For example, behaviour change techniques are used to set goals in health promotion interventions, and although these techniques provide a feasible framework to change behaviour, it is rather unknown how these techniques can be translated to design persuasive eHealth technologies.

- Understanding and predicting factors that improve adherence. For example, to understand what features matter most for whom, made possible, for instance, by using log data to observe real-time use of an eHealth technology. In future research, Artificial Intelligence (e.g., machine learning) can be used to optimize adherence via personalization of the technology because of knowledge on the patterns of usage, predicting non-usages and to create user-profiles.

- Identifying what features are most effective and in what combinations. Experimental research designs (see Chapter 14) can be used to investigate which (persuasive) features and which combinations within eHealth technology have most impact.

- Creating persuasive eHealth technologies that have a fit with the users. A promising approach is to design technologies that increase user engagement (see Chapter 13). Engagement may also provide an opportunity to personalize interventions: different people can be engaged by different persuasive features. By selecting only these features that invoke high engagement, for each individual the optimal intervention may be composed. 


\section{Key References}

Oinas-Kukkonen, H., \& Harjumaa, M. (2009). Persuasive systems design: Key issues, process model, and system features. Communications of the Association for Information Systems, 24(1), 28.

Oinas-Kukkonen, H. (2013). A foundation for the study of behavior change support systems. Personal and Ubiquitous Computing, 17(6), 1223-1235.

Kelders, S. M., Kok, R. N., Ossebaard, H. C., \& Van Gemert-Pijnen, J. E. (2012). Persuasive system design does matter: a systematic review of adherence to web-based interventions. Journal of Medical Internet Research, 14(6).

Jong, N. d., Wentzel, M., Kelders, S., Oinas-Kukkonen, H., \& Gemert-Pijnen, J. (2014). Evaluation of perceived persuasiveness constructs by combining user tests and expert assessments.

Fogg, B. J. (2002). Persuasive technology: using computers to change what we think and do. Ubiquity, 2002 (December), 5.

Hamari, J., Koivisto, J., \& Pakkanen, T. (2014). Do persuasive technologies persuade?-a review of empirical studies. Paper presented at the International Conference on Persuasive Technology.

\section{References}

Ajzen, I. (1991). The theory of planned behavior. Organizational behavior and human decision processes, 50(2), 179-211.

Ajzen, I. (1992). Persuasive communication theory in social psychology: A historical perspective. Influencing Human Behavior, 1-27.

Akkersdijk, S., Kelders, S., \& Gemert-Pijnen, J. (2016). The Grid, Classification of eHealth Applications Towards a Better (re) Design and Evaluation. Paper presented at the eTELEMED 2016: The Eighth International Conference on eHealth, Telemedicine, and Social Medicine.

Andrew, A., Borriello, G., \& Fogarty, J. (2007). Toward a systematic understanding of suggestion tactics in persuasive technologies. Persuasive Technology, 259-270.

Beerlage-de Jong, N., Eikelenboom-Boskamp, A., Voss, A., Sanderman, R., \& van GemertPijnen, J. (2014). Combining user-centered design with the persuasive systems design model; the development process of a web-based registration and monitoring system for healthcareassociated infections in nursing homes. International Journal on Advances in Life Sciences, 6, 262-271.

Beerlage-de Jong, N., Wrede, C., van Gemert-Pijnen, J., \& Sieverink, F. (2017). Storyboarding persuasion to match personality traits. Paper presented at the 12th International Conference on Perusasive Technology, Amsterdam, the Netherlands.

Bioethics, N. A. C. o. (2015). Nudging in Public Health - An Ethical Framework.

Cavanagh, K., \& Millings, A. (2013). (Inter) personal computing: the role of the therapeutic relationship in e-mental health. Journal of Contemporary Psychotherapy, 43(4), 197-206. 
Cialdini, R. B. (2001). Science and practice.

Consolvo, S., McDonald, D. W., \& Landay, J. A. (2009). Theory-driven design strategies for technologies that support behavior change in everyday life. Proceedings of the SIGCHI conference on human factors in computing systems.

Davis, F. D. (1985). A technology acceptance model for empirically testing new end-user information systems: Theory and results. Massachusetts Institute of Technology.

Dillard, J. P., \& Pfau, M. (2002). The persuasion handbook: Developments in theory and practice: Sage Publications.

Drozd, F., Lehto, T., \& Oinas-Kukkonen, H. (2012). Exploring perceived persuasiveness of a behavior change support system: a structural model. Persuasive Technology. Design for health and safety, 157-168.

Fishbein, M. (1979). A theory of reasoned action: some applications and implications.

Fogg, B. J. (2002). Persuasive technology: using computers to change what we think and do. Ubiquity, 2002(December), 5.

Freyne, J., Saunders, I., Brindal, E., Berkovsky, S., \& Smith, G. (2012). Factors associated with persistent participation in an online diet intervention. Paper presented at the CHI'12 Extended Abstracts on Human Factors in Computing Systems.

Halko, S., \& Kientz, J. (2010). Personality and persuasive technology: an exploratory study on health-promoting mobile applications. Persuasive technology, 150-161.

Hamari, J., Koivisto, J., \& Pakkanen, T. (2014). Do persuasive technologies persuade?-a review of empirical studies. Paper presented at the International Conference on Persuasive Technology.

IJsselsteijn, W., de Kort, Y., Midden, C., Eggen, B., \& van den Hoven, E. (2006). Persuasive technology for human well-being: setting the scene. Persuasive technology, 1-5.

Jong, N. d., Wentzel, M., Kelders, S., Oinas-Kukkonen, H., \& Gemert-Pijnen, J. (2014). Evaluation of perceived persuasiveness constructs by combining user tests and expert assessments.

Kaptein, M., Lacroix, J., \& Saini, P. (2010). Individual Differences in Persuadability in the Health Promotion Domain. PERSUASIVE, 6137, 94-105.

Kelders, S. M., Bohlmeijer, E. T., \& Van Gemert-Pijnen, J. E. (2013). Participants, usage, and use patterns of a web-based intervention for the prevention of depression within a randomized controlled trial. Journal of medical Internet research, 15(8).

Kelders, S. M., Kok, R. N., Ossebaard, H. C., \& Van Gemert-Pijnen, J. E. (2012). Persuasive system design does matter: a systematic review of adherence to web-based interventions. Journal of medical Internet research, 14(6).

Kelders, S. M., \& van Gemert-Pijnen, J. E. L. (2013). Using log-data as a starting point to make eHealth more persuasive. Paper presented at the International Conference on Persuasive Technology.

Kotler, P., Roberto, N., \& Lee, N. (2002). Social marketing: Improving the quality of life.

Kreps, G. L., Bonaguro, E. W., \& Query Jr, J. L. (2003). The history and development of the field of health communication. Russian Journal of Communication, 10, 12-20.

Kreps, G. L., \& Maibach, E. W. (2008). Transdisciplinary science: The nexus between communication and public health. Journal of Communication, 58(4), 732-748.

Langrial, S., Lehto, T., Oinas-Kukkonen, H., Harjumaa, M., \& Karppinen, P. (2012). Native Mobile Applications For Personal Well-Being: A Persuasive Systems Design Evaluation. Paper presented at the PACIS. 
Lehto, T., \& Oinas-Kukkonen, H. (2011). Persuasive features in web-based alcohol and smoking interventions: a systematic review of the literature. Journal of Medical Internet Research, 13(3).

Lehto, T., Oinas-Kukkonen, H., \& Drozd, F. (2012). Factors affecting perceived persuasiveness of a behavior change support system. International Conference on Information Systems (ICIS 2012), Orlando, Florida, December 16-19.

http://aisel.aisnet.org/icis2012/proceedings/HumanBehavior/18/.

Ludden, G. D., Kelders, S. M., \& Snippert, B. H. (2014). This is your life! Paper presented at the International Conference on Persuasive Technology.

Ludden, G. D., van Rompay, T. J., Kelders, S. M., \& van Gemert-Pijnen, J. E. (2015). How to increase reach and adherence of web-based interventions: a design research viewpoint. Journal of medical Internet research, 17(7).

McGuire, W. (1985). Attitudes and Attitude Change: Handbook of Social Psychology, Vol. 2, L Gardner and A Elliot: Newyork: Random House.

Nijland, N., van Gemert-Pijnen, J. E., Kelders, S. M., Brandenburg, B. J., \& Seydel, E. R. (2011). Factors influencing the use of a Web-based application for supporting the self-care of patients with type 2 diabetes: a longitudinal study. Journal of medical Internet research, 13(3).

Oinas-Kukkonen, H., \& Harjumaa, M. (2009). Persuasive systems design: Key issues, process model, and system features. Communications of the Association for Information Systems, 24(1), 28.

Oinas-Kukkonen, H. (2013). A foundation for the study of behavior change support systems. Personal and Ubiquitous Computing, 17(6), 1223-1235.

Orji, R., Vassileva, J., \& Mandryk, R. L. (2014). Modeling the efficacy of persuasive strategies for different gamer types in serious games for health. User Modeling and User-Adapted Interaction, 24(5), 453-498.

Perloff, R. M. (1993). Third-person effect research 1983-1992: A review and synthesis. International Journal of Public Opinion Research, 5(2), 167-184.

Petty, R. E., \& Cacioppo, J. T. (1986). The elaboration likelihood model of persuasion. Advances in experimental social psychology, 19, 123-205.

Ploderer, B., Reitberger, W., Oinas-Kukkonen, H., \& van Gemert-Pijnen, J. (2014). Social interaction and reflection for behaviour change: Springer.

Räisänen, T., Lehto, T., \& Oinas-Kukkonen, H. (2010). Practical findings from applying the PSD model for evaluating software design specifications. Persuasive technology, 185-192.

Rimal, R. N., \& Lapinski, M. K. (2009). Why health communication is important in public health. Bulletin of the World Health Organization, 87(4), 247-247a.

Scholten, M., Kelders, S., \& van Gemert-Pijnen, J. (in press). Self-guided web-based interventions: a scoping review on user needs and on the potential of embodied conversational agents to address them. Journal of medical Internet research.

Schulz von Thun, F. (1981). Miteinander reden 1. Störungen und Klärungen. Allgemeine Psychologie der Kommunikation: Rowohlt Taschenbuch Verlag, Reinbek bei Hamburg.

Sieverink, F., Kelders, S. M., Braakman-Jansen, L. M., \& van Gemert-Pijnen, J. E. (2014). The added value of $\log$ file analyses of the use of a personal health record for patients with type 2 diabetes mellitus: preliminary results. Journal of diabetes science and technology, 8(2), 247255. 
Van Gemert-Pijnen, J. E., Kelders, S. M., \& Bohlmeijer, E. T. (2014). Understanding the usage of content in a mental health intervention for depression: an analysis of log data. Journal of medical Internet research, 16(1).

Venkatesh, V., \& Davis, F. D. (2000). A theoretical extension of the technology acceptance model: Four longitudinal field studies. Management science, 46(2), 186-204.

Venkatesh, V., Morris, M. G., Davis, G. B., \& Davis, F. D. (2003). User acceptance of information technology: Toward a unified view. MIS quarterly, 425-478.

Wildeboer, G., Kelders, S. M., \& van Gemert-Pijnen, J. E. (2016). The relationship between persuasive technology principles, adherence and effect of web-Based interventions for mental health: A meta-analysis. International journal of medical informatics, 96, 71-85.

$\mathrm{Xu}$, A., Chomutare, T., \& Iyengar, S. (2014). Systematic review of behavioral obesity interventions and their persuasive qualities. Paper presented at the International Conference on Persuasive Technology. 